\title{
TRAZENDO A RIQUEZA ARBÓREA REGIONAL PARA DENTRO DAS CIDADES: POSSIBILIDADES, LIMITAÇÕES E BENEFÍCIOS ${ }^{1}$
}

\author{
Ingo Isernhagen ${ }^{2}$, Jeanne M.G. Le Bourlegat ${ }^{3}$, Marina Carboni ${ }^{2}$ \\ (recebido em 08.12.2008 e aceito para publicação em 21.05.2009)
}

\begin{abstract}
RESUMO
Apresenta-se uma apologia ao uso de espécies nativas regionais na silvicultura urbana das cidades. Parte-se da premissa que a conservação da diversidade biológica é hoje reconhecida como uma necessidade mundial, sendo preciso estender as estratégias de conservação para dentro do planejamento das áreas verdes das cidades. O uso dessas espécies arbóreas nativas regionais é hoje bastante incipiente, com nítido predomínio de espécies exóticas tanto no número de espécies como, principalmente, no número de árvores utilizadas. Ao utilizar espécies nativas regionais pode-se aumentar a permeabilidade da paisagem às demais espécies da biota, contribuindo para a conservação biológica regional. Os administradores dos espaços urbanos devem incentivar a realização de estudos para avaliar o uso das espécies arbóreas de ecossistemas regionais na arborização viária e na implantação de parques. Os autores entendem que, atendidos requisitos mínimos, não há razões para a não utilização dessas espécies, dados os grandes benefícios que podem trazer.
\end{abstract}

Palavras-Chave: árvores nativas regionais; silvicultura urbana; conservação da natureza; biodiversidade.

\footnotetext{
${ }^{1}$ Trabalho desenvolvido na disciplina Silvicultura Urbana (LCF5865), Prof. Demóstenes da Silva Filho, Programa de PósGraduação em Recursos Florestais, ESALQ, USP.

2 Biólogos, Doutorandos no Programa de Pós-Graduação em Recursos Florestais, ESALQ, USP - ingoise@gmail.com; marina@hospedaria.com.br; Rua Paulo Setúbal, 194 - Vila Independência - CEP 13418-393, Piracicaba/SP.

${ }^{3}$ Bióloga, Mestranda no Programa de Pós-Graduação em Recursos Florestais, ESALQ, USP - jeanne glb@yahoo.com.br.
} 


\title{
BRINGING REGIONAL TREE RICHNESS INTO THE CITIES: POSSIBILITIES, LIMITATIONS AND BENEFITS
}

\begin{abstract}
An apology to the use of regional native species in urban silviculture is presented. Considering biological diversity conservation as a worldwide need, it is necessary to extend conservation strategies to the green areas management in the cities. The use of these species is still incipient, with clear predominance of exotic species, considering both the number of species and, especially, of trees. By using regional native tree species it would be possible to increase landscape permeability to other regional species, improving regional biological conservation. To reach that goal, local urban managers should stimulate the number of studies to evaluate the use of regional native tree species in streets and parks planning. The authors understand that, reached the minimum requisites, there are no reasons not to use these species, considering the large benefits they could bring.
\end{abstract}

Keywords: regional native trees; urban silviculture; nature conservation; biodiversity. 


\section{A CONSERVAÇÃO DA NATUREZA NAS CIDADES}

Com o crescimento desordenado das cidades tornam-se nítidos os problemas associados, como poluição, falta de qualidade de serviços sanitários, deficiência de infraestrutura de transporte, problemas de saúde pública, entre outros. Nos países em desenvolvimento o crescimento populacional nos grandes centros urbanos é alarmante, especialmente nas periferias (MILLER, 1997), levando a conflitos no uso da terra que, em geral, acabam por gerar a supressão de espaços que poderiam tornar-se parques ou outros tipos de áreas verdes. Além da própria questão de perda de áreas verdes para usufruto humano, a urbanização reconhecidamente gera o declínio na riqueza de espécies nativas, a perda e fragmentação de habitats e a introdução de espécies exóticas. A convivência de algumas espécies com o ser humano, especialmente animais de grande porte, torna-se muitas vezes incompatível. Por outro lado, algumas espécies outrora inexistentes passam a ter espaço, especialmente aquelas mais adaptadas a locais alterados (MILLER, 1997), muitas delas exóticas. Mcdonald et al. (2008) apresentaram análises de cenários para o ano de 2030 , demonstrando impactos negativos potenciais sobre algumas espécies raras e ecoregiões em todo o planeta devido à expansão de áreas urbanas. Um desses impactos será a diminuição da distância entre as cidades e áreas protegidas, aumentando os riscos à conservação de espécies nesses locais.

Ao avaliar o impacto das cidades na paisagem, ocorre uma notória modificação do padrão regional. O padrão complexo e muitas vezes desordenado de crescimento da maior parte das cidades pode levar a um aumento da heterogeneidade dos padrões na paisagem. Do ponto de vista biológico a heterogeneidade espacial pode ser positiva, uma vez que cria mais habitats para a sobrevivência de espécies. No entanto, embora na malha urbana a paisagem possa ser mais heterogênea, nem sempre essa é positiva e amistosa para as espécies locais, uma vez que pode dificultar alguns processos biológicos naturais como deslocamentos, busca por alimentos e reprodução, podendo inclusive levar a extinções locais. Seriam então necessários locais que aumentassem a permeabilidade da matriz da paisagem, como fragmentos naturais, corredores ou trampolins ecológicos (FORMAN \& GODRON, 1986).

A incompatibilidade entre conservação da natureza e as cidades não é uma regra. Há registros antigos de convivência entre as construções e espaços verdes (FORMAN \& GODRON, 1986; MILLER, 1997). Desde a Conferência Rio-92, a conservação da biodiversidade passou a ser um dos principais pilares das estratégias globais de conservação da natureza (ZERBE, 2003), tendo também reflexos na gestão dos espaços urbanos. Essa diretriz global entra em sintonia com a tendência, especialmente em cidades norte-americanas e européias, de revitalização e valorização de espaços verdes urbanos 
(MILLER, 1997), na medida em que as pessoas buscam locais e momentos de descanso e recreação. Harris et al. (1999) reforçam que os remanescentes de vegetação nativa atingidos pela expansão urbana poderiam, dependendo do plano de urbanização, ser incorporados como parques urbanos. Esses parques, por sua vez, poderiam servir como zonas de transição entre o ambiente urbano consolidado e os remanescentes naturais preservados, a partir de um planejamento adequado de ocupação do espaço e da subseqüente fiscalização. O entorno dos parques urbanos também precisa de um adequado manejo, e nesse aspecto o Poder Público possui importância crucial no estabelecimento de normas urbanísticas e nas políticas de preservação ambiental (OLIVEIRA \& SANTOS, 2004).

Em artigo de revisão de Jim (2004), são apresentadas várias indicações de manejo dos espaços verdes urbanos, tanto para manter os já existentes como para novas alocações. Kenworthy (2006) também apresenta algumas discussões a respeito de como organizar o espaço nas "cidades-ecológicas", de forma a contemplar o acesso às áreas verdes e a distribuição dessas na malha urbana. Entre outras sugestões, o autor é favorável à concentração da malha urbana com um eficiente sistema de transporte público, de forma a minimizar o avanço da cidade sobre áreas verdes que podem, por sua vez, servir como parques urbanos ou para o fornecimento de alimento e outros bens. Sousa (2003) apresenta algumas iniciativas de readequação de espaços urbanos ociosos em áreas verdes, iniciativa que pode trazer benefícios que vão desde melhoria da qualidade do solo, criação de locais de recreação, revitalização econômica do bairro, entre outros. Outra idéia adotada em várias grandes cidades é a dos cinturões-verdes (MILLER, 1997), como o existente no entorno da cidade de São Paulo. Esses espaços podem contribuir na proteção da biodiversidade local, além de abrigar áreas de mananciais essenciais ao abastecimento da cidade, ajudar na estabilização climática, na conservação de solos e na garantia de segurança alimentar para as cidades (INSTITUTO FLORESTAL, 2008).

\section{O USO DAS ÁRVORES NATIVAS REGIONAIS}

Considerando a relevância e a intenção de conservar a biodiversidade em espaços urbanos, é importante envolver na discussão a importância do uso de espécies nativas. Biondi \& Leal (2008) apresentam uma revisão relevante de trabalhos que indicaram a importância e as vantagens do uso de espécies nativas na arborização urbana, como maior resistência a pragas, a criação de um banco genético ex-situ, a minimização do risco de uso de espécies exóticas invasoras, etc. Reis et al. (2003) sugerem que, ao formar conjuntos que lembrem a paisagem original da região, seria possível criar uma nova percepção do espaço urbano por parte dos habitantes, contribuindo na re-educação para a valorização da 
biodiversidade no ambiente urbano. Os mesmos autores também ressaltam a importância do uso de nativas nas áreas urbanas como uma forma de conservação ex situ, sendo para isso importante a produção de mudas com alta variabilidade genética, opinião compartilhada por Clarkson et al. (2007). Nesse sentido, ganha importância também o uso de espécies nativas em processo de extinção como uma forma complementar de conservação da natureza.

Alvey (2007) apresenta o termo "homogenização biótica" para o fenômeno de substituição das espécies nativas por exóticas em ecossistemas urbanos. Esse fenômeno é associado à escolha das espécies e também às alterações das condições ambientais locais que, por sua vez, passam a não ser mais apropriadas às espécies nativas. O mesmo autor, que no trabalho desenvolve uma revisão sobre o tema promoção de biodiversidade em florestas urbanas, relata que apesar da diversidade local poder aumentar com a introdução de espécies exóticas, a diversidade em uma escala global diminui com essa escolha, e a incidência de pragas em áreas com menor diversidade é mais devastadora.

Brun et al. (2007) apresentam uma revisão sobre os benefícios da arborização urbana para manutenção da diversidade da fauna. Espécies animais nativas dos arredores urbanos podem beneficiar-se com as fontes de abrigo e alimento geradas pelas árvores urbanas, especialmente quando essas forem nativas regionais. Dados revisados por Reis et al. (2003) demonstram que a oferta de recursos (flores e frutos) proporcionada por árvores nativas para a fauna é pronunciada, ocorrendo ao longo de todo ano e propiciando fortes níveis de interação com a fauna regional.

Essas árvores nativas, juntamente com praças e parques, podem também funcionar como corredores ecológicos. Pennignton et al. (2008), ao analisarem as preferências de habitats de aves migratórias em áreas urbanas, relataram que estas tendem a escolher áreas verdes maiores, especialmente as áreas ao longo de rios. Aves neotropicais migratórias em trânsito preferiram usar árvores nativas e mais maduras, muito possivelmente devido à maior oferta de recursos alimentares. Garden et al. (2007) indicaram que, ao se planejar as melhores formas de manejo para conservação da fauna nativa nas cidades, é preciso fazer um balanço da auto-ecologia dessas espécies, permitindo uma ação mais integrada que conserve o maior número possível de espécies.

Dada a riqueza de formações fitogeográficas encontradas no Brasil, é preciso especificar melhor em qual dessas formações a espécie é nativa. Ao se generalizar o uso do termo "nativa" pode-se incorrer no risco de atribuir as mesmas características ecológicas a uma espécie da Floresta Amazônica e uma espécie de Cerrado ou Caatinga. Mesmo dentro de uma mesma formação fitogeográfica podem ocorrer variações genotípicas entre populações de uma mesma espécie, relacionadas a especializações desenvolvidas ao longo das gerações, o que caracteriza os chamados ecótipos. Por outro lado, outras espécies 
podem demonstrar maior plasticidade funcional, ocorrendo em mais de um ecossistema, com um potencial interessante para uso em arborização urbana. Blum et al. (2008) usam o termo "espécies nativas" para aquelas de ocorrência natural da região fitogeográfica onde se insere a cidade ou local em avaliação. No presente trabalho, apresenta-se a proposta de denominar essas espécies de "nativas regionais", enquanto as "nativas" seriam aquelas encontradas em outros ecossistemas brasileiros exceto o da região em estudo, e "exóticas" para aquelas de ecossistemas não encontrados no Brasil. Ao pensarmos em características adaptativas e no fato de que a natureza não segue padrões geográficos baseados em fronteiras políticas, as espécies não nativas da região, mas pertencentes a outros ecossistemas dentro do Brasil, poderiam também ser consideradas exóticas, mas no presente trabalho essas categorias foram mantidas divididas. Essa classificação, embora possa ser encarada como um preciosismo, é importante na medida em que toda a biota associada na região está evolutivamente inter-relacionada. Ao se utilizarem as espécies nativas regionais na arborização urbana, a coexistência e sobrevivência dessas espécies em escala local poderiam ser garantidas. O termo "nativa regional" não é novo, mas ainda não é muito difundido.

É muito comum listas de árvores nativas utilizadas nos municípios apresentarem espécies não regionais, de outros ecossistemas. Assim, o uso do termo "nativa" deve ser utilizado e lido com cautela e critério, como já alertado anteriormente. Da mesma forma, a divulgação de altos índices de riqueza ou diversidade de espécies na arborização urbana devem ser analisados criteriosamente, pois podem mascarar a presença de espécies exóticas e nativas de outros ecossistemas que não os da região avaliada. Um índice de diversidade de espécies elevado pode não necessariamente significar qualidade ambiental para a biota regional. Porém, para que as espécies arbóreas nativas regionais possam ser utilizadas na arborização urbana, cabe uma avaliação sobre o potencial para atender aos critérios utilizados na escolha de árvores a serem utilizadas nas cidades.

Os vários benefícios da arborização das ruas e avenidas estão condicionados à qualidade de seu planejamento, visto que muitos fatores podem interferir no desenvolvimento das árvores na área urbana. Entre esses fatores citam-se a compactação e impermeabilização do solo devido à pavimentação, poluição do ar, que impede a folha de exercer livremente suas funções, podas drásticas e abertura de valas junto à árvore, mutilando o seu sistema radicular (PIVETTA \& SILVA FILHO, 2002). A presença de espécies inadequadas na zona urbana também pode ocasionar graves problemas como, por exemplo, curto-circuito gerado pelo contato dos galhos com fiação elétrica nua, ou a danificação dos sistemas de água esgoto, telefone e gás de subsolo pelo crescimento de raízes superficiais (MARTO et al., 2006). Para um adequado planejamento da arborização das ruas e avenidas devem-se conhecer as características particulares de cada espécie, 
bem como seu comportamento nas condições ambientais a que serão impostas (PIVETTA \& SILVA FILHO, 2002). Este é o principal desafio para a utilização de árvores nativas regionais na arborização urbana: a falta de conhecimento das características destas espécies e do seu comportamento neste ambiente desfavorável. Nem sempre as espécies nativas apresentam um desempenho tão bom quanto as exóticas. Ao escolher a espécie é necessário avaliar a adaptabilidade dessas ao ambiente grandemente modificado (HARRIS et al., 1999).

De acordo com Marto et al. (2006) há uma série de características que podem ser avaliadas durante a seleção das espécies, como a tolerância a poluentes e às baixas condições de aeração do solo, presença de odores, tempo de crescimento e de longevidade, tamanho e cor das flores e frutos, época e duração do florescimento e frutificação, entre outros. Dentre estas características, Lazaro et al. (2002), Marto et al. (2006), Pivetta e Silva Filho (2002), Crestana et al. (2007), listaram algumas desejáveis (embora discutíveis na opinião dos autores do presente trabalho) para a definição das espécies nativas a serem utilizadas na arborização urbana, como por exemplo: porte adequado ao espaço disponível; rusticidade; desenvolvimento rápido; resistência a pragas e doenças; sistema radicular profundo e pivotante; tronco único, sem espinhos e copa bem definida; tronco e ramos consistentes; folhas pequenas, não coriáceas e não caducifólias; folhas pilosas e cerosas; inflorescências grandes e densas com flores pequenas, pouco suculentas e com cores vivas; frutos pequenos e silvestres; ausência de toxicidade e princípios alergênicos.

Já existem também publicações recentes com listagem de espécies nativas de cada Bioma, indicadas para uso na arborização urbana (LAZARO et al., 2002; PIVETTA \& SILVA FILHO, 2002; MACHADO et al., 2006). Porém, o número de guias dessa natureza ainda está muito aquém do desejável.

\section{BALANÇO DO USO DE ÁRVORES NATIVAS REGIONAIS NO BRASIL}

Apesar de se observar nas cidades brasileiras uma nítida predominância do uso de espécies exóticas sobre as nativas, existem algumas exceções. Em estudos realizados em Nova Iguaçu (RJ) (ROCHA et al., 2004), em Águas de São Pedro (SP) (SILVA FILHO \& BORTOLETO, 2005) e também em Teresina (PI) (MACHADO et al., 2006), foi encontrado grande número de espécies nativas, embora muitas vezes tenham ocorrido problemas de adequação ao local e ao método de avaliação da qualidade da arborização de ruas. No Estado de São Paulo, Bortoleto et al. (2007) encontraram 38,67\% de espécies nativas na arborização viária da Estância Turística de Águas de São Pedro. Faria et al. (2007), em 
levantamento florístico da arborização urbana de Jacareí - SP, encontraram 50\% de espécies nativas, sendo que das 14 espécies nativas, parte são frutíferas, plantadas pela população. Rossato et al. (2008) encontraram 23 espécies nativas, que representam 31,18\% das espécies da arborização da cidade de Assis (SP). Em Campina Grande (PB), Dantas e Souza (2004) encontraram 65 espécies nativas, que representam 48,8\% do total de espécies da arborização urbana. Pires et al. (2007) encontraram para a cidade de Goiandira (GO), um total de $10 \%$ de espécies nativas regionais na cidade. De acordo com Coletto et al. (2008), das 60 espécies encontradas no Município de Sete de Setembro (RS), 24 (40\%) são nativas. Em Terezina (PI), no levantamento de espécies nativas realizado por MACHADO et al. (2006), foram observadas 48 espécies, distribuídas em 42 gêneros e 21 famílias botânicas, número considerado bom pelos autores.

Os inventários de arborização urbana municipal, entre seus vários benefícios, são excelentes ferramentas para diagnóstico da proporção entre espécies exóticas, nativas e nativas regionais, desde que interpretadas conforme a proposta do presente trabalho. Silva (2008) indica que a maioria dos censos de arborização disponível em publicações revela o predomínio de espécies que não fazem parte do bioma local, e várias dessas tem potencial invasor. A mesma autora sugere que o fato de cidades não possuírem identidade arbórea local, usando predominantemente espécies exóticas comuns, pode inclusive afastar turistas que se interessariam por peculiaridades locais. Ainda segundo a autora, os biomas brasileiros são suficientemente ricos em espécies, faltando mais estudos sobre a adaptabilidade dessas árvores às condições locais. Em uma análise expedita, pode-se comprovar que a maior parte dos inventários realizados no Brasil, inclusive alguns supracitados, não especificam quais espécies nativas são regionais.

A título de amostragem para a avaliação da proporção de uso de espécies das três ocorrências citadas no presente trabalho, foram feitas breves análises de dados de inventários feitos em três municípios do Estado de São Paulo: Jaú (a partir de SOUZA et al., 2004), Bocaina (a partir de MARQUES, 2005) e Piracicaba (a partir de Demóstenes da Silva Filho, dados não publicados).

Para o inventário do município de Jaú, realizado entre 2003-2004, o município foi dividido em sete zonas. Foi feito um plano de amostragem de 15\% do número total de amostras possíveis em cada zona, para determinar o número de amostras necessárias visando ter um erro de no máximo de 10\%, com 95\% de probabilidade, uma quantidade estatística e economicamente aceitável para o trabalho em questão. Para a classificação da ocorrência das espécies, embora tenham sido consideradas as informações do trabalho original, a lista foi revisada utilizando critérios simplificados e adaptados de Blum et al. (2008). Dessa forma, consideram-se três categorias: nativas regionais (de ocorrência na Floresta Estacional Semidecidual da região de Jaú), nativas brasileiras (presentes em outras 
regiões fitogeográficas encontradas no Brasil, como Savana e Floresta Ombrófila Densa, por exemplo) e exóticas (não encontradas em ecossistemas brasileiros). A verificação de sinonímias e a indicação da ocorrência das espécies foram baseadas em Lorenzi (1992, 1998) e MOBOT (2008), e as famílias foram organizadas segundo sistema APG II (SOUZA \& LORENZI, 2005). A Tabela 1 apresenta as espécies encontradas.

Foram encontradas 75 espécies no levantamento realizado, número considerado baixo pelos próprios responsáveis pela pesquisa em função da riqueza de espécies de árvores registrada na região de Jaú: 145 espécies arbustivo-arbóreas, segundo levantamento de Nicolini (1990). Na realidade, esse número é ainda menos representativo quando se considera a proporção de espécies nativas regionais: das 75 espécies amostradas 48 (64\%) são exóticas, 10 (13,33\%) são nativas de outros ecossistemas brasileiros diferentes dos encontrados na região e 17 (22,67\%) são nativas regionais. Entre as famílias com maior número de espécies estão Fabaceae, com 16 (21,33\%), Bignoniaceae, com $8(10,66 \%)$ e Rutaceae, com 5 (6,66\%), tendo sido encontradas, ao todo, espécies de 30 famílias botânicas.

Tabela 1: Espécies encontradas no levantamento arbóreo de Jaú - SP (organizadas em ordem alfabética de nome popular).

\begin{tabular}{|c|c|c|c|}
\hline Nome cientifico & Família & Nome popular & Ocorrência \\
\hline Annona sp. & Annonaceae & Fruta do conde & Exótica \\
\hline Averrhoa carambola L. & Oxalidaceae & Carambola & Exótica \\
\hline Bauhinia variegata L. & Fabaceae & Pata-de-vaca & Exótica \\
\hline Brunfelsia uniflora (Pohl) D. Don & Solanaceae & Manacá-de-cheiro & Exótica \\
\hline Caesalpinia pucherrima (L.) Sw. & Fabaceae & Flamboyant mirim & Exótica \\
\hline Cajanus cajan (L.) Millsp. & Fabaceae & Feijão guandu & Exótica \\
\hline Carica papaya L. & Caricaceae & Mamoeiro & Exótica \\
\hline Cassia fistula L. & Fabaceae & Cássia imperial & Exótica \\
\hline Chamaecyparis sp. & Cupressaceae & Pinheiro & Exótica \\
\hline Cinnamomum zeylanicum Blume & Lauraceae & Canela & Exótica \\
\hline Citrus limon (L.) Burm. f. & Rutaceae & Limoeiro & Exótica \\
\hline Citrus sp1. & Rutaceae & Laranja cravo & Exótica \\
\hline Citrus sp2. & Rutaceae & Laranjeira & Exótica \\
\hline Citrus sp3. & Rutaceae & Pôncirus & Exótica \\
\hline $\begin{array}{l}\text { Codiaeum variegatum (L.) Rumph. ex A. } \\
\text { Juss. }\end{array}$ & Euphorbiaceae & Croton & Exótica \\
\hline Delonix regia (Bojer ex Hook.) Raf. & Fabaceae & Flamboyant & Exótica \\
\hline Dombeya wallichii (Lindl.) k. Schum. & Malvaceae & Astrapeia & Exótica \\
\hline Duranta repens L. & Verbenaceae & Violeteira & Exótica \\
\hline Eriobotrya japonica (Thunb.) Lindl. & Rosaceae & Nêspera & Exótica \\
\hline Ficus benjamina L. & Moraceae & Ficus & Exótica \\
\hline Fuchsia sp. & Onagraceae & Brinco de princesa & Exótica \\
\hline Grevillea banksii R.Br. & Proteaceae & Grevilha anã & Exótica \\
\hline Hibiscus rosa-sinensis L. & Malvaceae & Hibisco & Exótica \\
\hline Jacaranda mimosifolia D.Don & Bignoniaceae & Jacarandá mimoso & Exótica \\
\hline Lagerstroemia indica L. & Lythraceae & Resedá & Exótica \\
\hline Leucaena leucocephala (Lam.) de Wit & Fabaceae & Leucena & Exótica \\
\hline Ligustrum lucidum W.T. Aiton & Oleaceae & Legustre & Exótica \\
\hline Macadamia integrifolia Maiden \& Betche & Proteaceae & Macadâmia & Exótica \\
\hline Magnolia champaca (L.) Baill. ex Pierre & Magnoliaceae & Magnólia & Exótica \\
\hline Malpighia glabra L. & Malpighiaceae & Acerola & Exótica \\
\hline Mangifera indica $\mathrm{L}$. & Anacardiaceae & Mangueira & Exótica \\
\hline
\end{tabular}




\begin{tabular}{|c|c|c|c|}
\hline Melia azedarach L. & Meliaceae & Santa bárbara & Exótica \\
\hline Morus nigra L. & Moraceae & Amora & Exótica \\
\hline Murraya paniculata (L.) Jack & Rutaceae & Murta & Exótica \\
\hline Nerium oleander L. & Apocynaceae & Espirradeira & Exótica \\
\hline Persea americana Mill. & Lauraceae & Abacateiro & Exótica \\
\hline Plumeria rubra L. & Apocynaceae & Jasmim-manga & Exótica \\
\hline Prunus domestica L. & Rosaceae & Ameixa & Exótica \\
\hline Prunus sp. & Rosaceae & Pessegueiro & Exótica \\
\hline Punica granatum $\mathrm{L}$. & Lythraceae & Romã & Exótica \\
\hline Nome cientifico & Família & Nome popular & Ocorrência \\
\hline Schefflera actinophylla (Endl.) Harms & Araliaceae & Cheflera & Exótica \\
\hline Senna bicapsularis L. Roxb. & Fabaceae & Canudo de pito & Exótica \\
\hline Spathodea nilotica Seem. & Bignoniaceae & Espatódia & Exótica \\
\hline Syzygium cumini (L.) Skeels & Myrtaceae & Jambolão & Exótica \\
\hline Tecoma stans (L.) Juss. ex Kunth & Bignoniaceae & Ipê-de-jardim & Exótica \\
\hline Terminalia catappa L. & Combretaceae & Chapéu-de-sol & Exótica \\
\hline Thevetia peruviana (Pers.) K. Schum. & Apocynaceae & Chapéu-de-napoleão & Exótica \\
\hline Tipuana tipu (Benth.) Kuntze & Fabaceae & Tipuana & Exótica \\
\hline Caesalpinia echinata Lam. & Fabaceae & Pau-brasil & Nativa \\
\hline Caesalpinia peltophoroides Benth. & Fabaceae & Sibipiruna & Nativa \\
\hline Licania tomentosa (Benth.) Fritsch & Chrysobalanaceae & Oiti & Nativa \\
\hline Lophanthera lactescens Ducke & Malpighiaceae & $\begin{array}{r}\text { Lofantera da } \\
\text { Amazônia }\end{array}$ & Nativa \\
\hline Pachira aquatica Aubl. & Malvaceae & Monguba & Nativa \\
\hline Psidium sp. & Myrtaceae & Goiabeira & Nativa \\
\hline Schinus molle L. & Anacardiaceae & Chorão & Nativa \\
\hline Schinus terebinthifolia Raddi & Anacardiaceae & Aroeira pimenteira & Nativa \\
\hline Tibouchina granulosa (Desr.) Cogn. & Melastomataceae & Quaresmeira & Nativa \\
\hline Tibouchina mutabilis Cong. & Melastomataceae & Manacá-da-serra & Nativa \\
\hline Albizia niopoides (Spruce ex Benth.) Burkart & Fabaceae & Farinha seca & Nativa Regional \\
\hline Cordia superba Cham. & Boraginaceae & Babosa branca & Nativa Regional \\
\hline Cordia trichotoma (Vell.) Arráb. ex Steud. & Boraginaceae & Louro pardo & Nativa Regional \\
\hline Eugenia uniflora L. & Myrtaceae & Pitanga & Nativa Regional \\
\hline Ficus guaranitica Chodat & Moraceae & Figueira branca & Nativa Regional \\
\hline Holocalyx balansae Micheli. & Fabaceae & Alecrim de campinas & Nativa Regional \\
\hline Myrciaria cauliflora (Mart.) O. Berg. & Myrtaceae & Jabuticaba & Nativa Regional \\
\hline Myroxylon peruiferum L.f. & Fabaceae & Cabreúva & Nativa Regional \\
\hline Nectandra megapotamica (Spreng.) Mez & Lauraceae & Canelinha & Nativa Regional \\
\hline Peltophorum dubium (Spreng.) Taub. & Fabaceae & Canafistula & Nativa Regional \\
\hline Platypodium elegans Vogel & Fabaceae & Amendoinzeiro & Nativa Regional \\
\hline $\begin{array}{l}\text { Senna macranthera (DC. ex Collad.) H.S. } \\
\text { Irwin \& Barneby }\end{array}$ & Fabaceae & Fedegoso & Nativa Regional \\
\hline Syagrus romanzoffiana (Cham.) Glassman & Arecaceae & Jerivá & Nativa Regional \\
\hline Tabebuia avellanedae Lorentz ex Griseb. & Bignoniaceae & Ipê rosa & Nativa Regional \\
\hline Tabebuia roseoalba (Ridl.) Sandwith & Bignoniaceae & Ipê branco & Nativa Regional \\
\hline Tabebuia serratifolia (Vahl) G. Nicholson & Bignoniaceae & Ipê amarelo & Nativa Regional \\
\hline Tabernaemontana fuchsiaefolia A. DC. & Apocynaceae & Leiteiro & Nativa Regional \\
\hline
\end{tabular}

No município de Bocaina, também no Estado de São Paulo, as espécies encontradas em censo realizado nos 169 quarteirões da cidade em 2005 estão listadas na Tabela 2. A verificação de sinonímias e a indicação da ocorrência das espécies foram baseadas em Lorenzi $(1992,1998)$ e MOBOT (2008), e as famílias foram organizadas segundo sistema APG II (SOUZA \& LORENZI, 2005). Ao todo foram encontradas 60 espécies, sendo 26 exóticas (43,34\%), 16 nativas de outros ecossistemas brasileiros (26,66\%) e 18 nativas regionais (30\%). No trabalho original ocorreu também uma divisão das espécies conforme as 3 categorias (nativa, nativa regional e exótica), sendo as nativas regionais denominadas como de "ocorrência natural na região central do estado de São Paulo". Mesmo assim, 
houve uma revisão da listagem conforme método utilizado para a listagem presente na Tabela 1. Novamente, entre as famílias, Fabaceae destacou-se em número de espécies (10spp, 16,66\%), seguida de Bignoniaceae (5 ssp., 8,33\%) e Myrtaceae (4spp, 6,66\%), com um total de 30 famílias representadas.

Tabela 2: Espécies encontradas no levantamento da arborização urbana de Bocaina - SP (organizadas conforme ocorrência: exótica, nativa ou nativa regional (ver texto)).

\begin{tabular}{|c|c|c|c|}
\hline Espécie & Família & Nome popular & Ocorrência \\
\hline Artocarpus integrifolia L. f. & Moraceae & Jaca & Exótica \\
\hline $\begin{array}{l}\text { Brugmansia suaveolens (Humb. \& Bonpl. ex } \\
\text { Willd.) Bercht. \& C. Presl }\end{array}$ & Solanaceae & Copo de leite & Exótica \\
\hline Caesalpinia pucherrima (L.) Sw. & Fabaceae & Flamboyant Mirim & Exótica \\
\hline Carica papaya L. & Caricaceae & Mamão & Exótica \\
\hline Cassia fistula L. & Fabaceae & Cássia Imperial & Exótica \\
\hline Citrus limon (L.) Burm. f. & Rutaceae & Limão & Exótica \\
\hline Codiaeum variegatum (L.) Rumph. ex A. Juss. & Euphorbiaceae & Loro variegado & Exótica \\
\hline Cordia abyssinica R. Br. & Boraginaceae & Córdia africana & Exótica \\
\hline Cupressus Iusitanica Mill. & Pinaceae & Cipreste & Exótica \\
\hline Delonix regia (Bojer ex Hook.) Raf. & Fabaceae & Flamboyant & Exótica \\
\hline Filicium decipiens (Wight \& Arn.) Thwaites & Sapindaceae & Manga & Exótica \\
\hline Glycine hispida (Moench) Maxim. & Fabaceae & Soja & Exótica \\
\hline Grevillea robusta A. Cunn. ex R. Br. & Proteaceae & Grevilha & Exótica \\
\hline Hibiscus rosa-sinensis L. & Malvaceae & Hibisco / Mimo & Exótica \\
\hline Ligustrum japonicum Thunb. & Oleaceae & Alfineiro ou Legustre & Exótica \\
\hline Magnolia grandiflora L. & Magnoliaceae & Magnólia & Exótica \\
\hline Malpighia glabra L. & Malpighiaceae & Acerola & Exótica \\
\hline Murraya paniculata (L.) Jack & Rutaceae & Murta & Exótica \\
\hline Nerium oleander $\mathrm{L}$. & Apocynaceae & Ficus benjamina & Exótica \\
\hline Osmanthus fragrans (Thunb.) Lo & Oleaceae & Jasmim & Exótica \\
\hline Punica granatum L. & Lythraceae & Romã & Exótica \\
\hline Spathodea nilotica Seem. & Bignoniaceae & Espatódia & Exótica \\
\hline Stryphnodendron adstringens (Mart.) Coville & Fabaceae & Barba de bode & Exótica \\
\hline Syzygium cumini (L.) Skeels & Myrtaceae & Jambolão & Exótica \\
\hline Terminalia catappa L. & Combretaceae & $\begin{array}{r}\text { Chapéu de Padre ou } \\
\text { de Praia }\end{array}$ & Exótica \\
\hline Thevetia thevetia (L.) Millsp. & Apocynaceae & Chapéu de Napoleão & Exótica \\
\hline Anacardium occidentale L. & Anacardiaceae & Caju & Nativa \\
\hline Araucaria angustifolia (Bertol.) Kuntze & Araucariaceae & Pinheiro-do-paraná & Nativa \\
\hline Bombacopsis glabra (Pasq.) Robyns & Bombacaceae & Castanha & Nativa \\
\hline Caesalpinia peltophoroides Benth. & Fabaceae & Sibipiruna & Nativa \\
\hline Caesalpinia echinata Lam. & Fabaceae & Pau Brasil & Nativa \\
\hline Couroupita guianensis Aubl. & Lecythidaceae & Abricó & Nativa \\
\hline Duranta repens L. & Verbenaceae & Pingo de Ouro & Nativa \\
\hline Hevea brasiliensis (Willd. ex A. Juss.) Müll. Arg. & Euphorbiaceae & Seringueira & Nativa \\
\hline Licania tomentosa (Benth.) Fritsch & Chrysobalanaceae & Oiti & Nativa \\
\hline Pachira aquatica Aubl. & Malvaceae & Monguba & Nativa \\
\hline Paullinia cupana Kunth & Sapindaceae & Guaraná & Nativa \\
\hline Physocalymma scaberrimum Pohl & Lythraceae & Resedá & Nativa \\
\hline Pollinia villosa Spreng. & Poaceae & Fruta do Conde & Nativa \\
\hline Tabebuia roseoalba (Ridl.) Sandwith & Bignoniaceae & Ipê Branco & Nativa \\
\hline Tecoma stans (L.) Juss. ex Kunth & Bignoniaceae & Ipê de Jardim & Nativa \\
\hline Theobroma cacao L. & Malvaceae & Cacau & Nativa \\
\hline Acrocomia aculeata (Jacq.) Lodd. ex Mart. & Arecaceae & Macaúva & Nativa Regional \\
\hline Attalea dubia (Mart.) Burret & Arecaceae & Palmeira-indaiá & Nativa Regional \\
\hline Bauhinia forficata Link & Fabaceae & Pata de Vaca & Nativa Regional \\
\hline Cedrela fissilis Vell. & Meliaceae & Cedro & Nativa Regional \\
\hline $\begin{array}{l}\text { Campomanesia guazumifolia (Cambess.) O. } \\
\text { Berg }\end{array}$ & Myrtaceae & Sete Capas & Nativa Regional \\
\hline Erythrina falcata Benth. & Fabaceae & Bico de papagaio & Nativa Regional \\
\hline Esenbeckia febrifuga (A. St.-Hil.) A. Juss. ex & Rutaceae & Mamona & Nativa Regional \\
\hline
\end{tabular}


ISERNHAGEN, I. ET AL.

\begin{tabular}{l|r|r|r}
\hline Mart. & & & \\
\hline Eugenia uniflora L. & Myrtaceae & Pitanga & Nativa Regional \\
\hline Holocalyx balansae Micheli. & Fabaceae & Alecrim de Campinas & Nativa Regional \\
\hline Nectandra megapotamica (Spreng.) Mez & Lauraceae & Canelinha & Nativa Regional \\
\hline Persea pyrifolia Nees & Lauraceae & Abacateiro-do-mato & Nativa Regional \\
\hline Psidium guajava L. & Myrtaceae & Goiabeira & Nativa Regional \\
\hline Sapium glandulatum (Vell.) Pax $\quad$ Espécie & Euphorbiaceae & Leiteiro & Nativa Regional \\
\hline \multicolumn{1}{|c|}{ Schinus molle L. } & Família & Nome popular & Ocorrência \\
\hline Schinus polygama (Cav.) Cabrera & Anacardiaceae & Aroeira Salsa & Nativa Regional \\
\hline Tabebuia alba (Cham.) Sandwith & Bignoniaceae & Coquinho & Nativa Regional \\
\hline Tabebuia avellanedae Lorentz ex Griseb. & Bignoniaceae & Ipê Amarelo & Nativa Regional \\
\hline Tibouchina granulosa (Desr.) Cogn. & Melastomataceae & Ipê Roxo & Nativa Regional \\
\hline
\end{tabular}

Já no município de Piracicaba foi realizado um levantamento entre os anos de 20032006, baseando os resultados em dados de amostragem de 32 bairros da cidade, sendo que em cada bairro foram amostradas 10 quadras. A verificação de sinonímias e a indicação da ocorrência das espécies foram baseadas em Lorenzi (1992, 1998), MOBOT (2008) e Rodrigues (1999), e as famílias foram organizadas segundo sistema APG II (SOUZA \& LORENZI, 2005). Das 153 espécies encontradas (Tabela 3), 92 eram exóticas (60,13\%), 28 nativas brasileiras (18,30\%) e 33 nativas regionais (21,57\%) (Figura 3). À semelhança de Jaú e Bocaina, Fabaceae foi a família com maior número de espécies (32spp., 20,92\%), seguida de Myrtaceae (16spp., 10,46\%) e Bignoniaceae (12spp., 7,84\%), com um total de 45 famílias encontradas.

Tabela 3: Espécies encontradas no levantamento da arborização urbana de Piracicaba - SP (organizadas conforme ocorrência: exótica, nativa ou nativa regional (ver texto)).

\begin{tabular}{l|r|r|r}
\multicolumn{1}{c|}{ Espécie } & Família & Nome Comum & Ocorrência \\
\hline Acacia podalyrifolia A. Cunn. ex G. Don & Fabaceae & Acácia-mimosa & Exótica \\
\hline Acer pseudoplatanus L. & Arecaceae & Plátano & Exótica \\
\hline Annona squamosa L. & Annonaceae & Exótica \\
\hline Araucaria columnaris Hook. & Pinaceae & Araucárria colunar & Exótica \\
\hline Areca catechu L. & Arecaceae & Palmeira betel & Exótica \\
\hline Averrhoa carambola L. & Oxalidaceae & Carambola & Exótica \\
\hline Bauhinia blakeana Dunn & Fabaceae & Pata-de-vaca-rosa & Exótica \\
\hline Bauhinia variegata L. & Fabaceae & Pata-de-vaca & Exótica \\
\hline $\begin{array}{l}\text { Bauhinia variegata var. candida (Aiton) Buch.- } \\
\text { Ham. }\end{array}$ & Fabaceae & Pata-de-vaca-branca & Exótica \\
\hline Bougainvillea spectabilis Willd. & Nyctaginaceae & Primavera & Exótica \\
\hline Brunfelsia uniflora (Pohl) D. Don & Solanaceae & Manacá-de-cheiro & Exótica \\
\hline Buddleja davidii Franch.. & Scrophulariaceae & Lilás-da-china & Exótica \\
\hline Caesalpinia pucherrima (L.) Sw. & Fabaceae & Flamboyant-de-jardim & Exótica \\
\hline Caesalpinia spinosa (Molina) Kuntze & Fabaceae & Falso pau-brasil & Exótica \\
\hline Cajanus cajan (L.) Millsp. & Fabaceae & Feijão-guandu & Exótica \\
\hline Callistemon speciosus (Sims) DC. & Myrtaceae & Escovinha-de-garrafa & Exótica \\
\hline Camellia japonica L. & Theaceae & Camélia & Exótica \\
\hline Carica papaya L. & Caricaceae & Mamoeiro & Exótica \\
\hline Caryota urens L. & Arecaceae & Palmeira rabo-de- & Exótica \\
\hline
\end{tabular}


Trazendo a riqueza arbórea regional...

\begin{tabular}{|c|c|c|c|}
\hline & & peixe & \\
\hline Cassia fistula $\mathrm{L}$. & Fabaceae & Chuva-de-ouro & Exótica \\
\hline Cassia grandis L. f. & Fabaceae & Cassia-rosa & Exótica \\
\hline Cestrum nocturnum $\mathrm{L}$. & Solanaceae & Dama-da-noite & Exótica \\
\hline Cinnamomum zeylanicum Blume & Lauraceae & Canela & Exótica \\
\hline Citrus aurantium $\mathrm{L}$. & Rutaceae & Laranjeira & Exótica \\
\hline Citrus limon (L.) Burm. f. & Rutaceae & Limoeiro & Exótica \\
\hline Espécie & Família & Nome Comum & Ocorrência \\
\hline Codiaeum variegatum (L.) Rumph. ex A. Juss. & Euphorbiaceae & Cróton & Exótica \\
\hline Coffea arabica L. & Rutaceae & Cafeeiro & Exótica \\
\hline Cryptomeria japonica (Thunb. ex L. f.) D. Don & Pinaceae & Criptoméria & Exótica \\
\hline Cupressus sempervirens L. & Pinaceae & $\begin{array}{r}\text { Cipreste-do- } \\
\text { mediterrâneo }\end{array}$ & Exótica \\
\hline Cupressus sp. & Pinaceae & Cipreste & Exótica \\
\hline Delonix regia (Bojer ex Hook.) Raf. & Fabaceae & Flamboyant & Exótica \\
\hline Duranta repens L. & Verbenaceae & Pingo de ouro & Exótica \\
\hline $\begin{array}{l}\text { Dypsis lutescens (H. Wendl.) Beentje \& J. } \\
\text { Dransf. }\end{array}$ & Arecaceae & Areca bambu & Exótica \\
\hline Eriobotrya japonica (Thunb.) Lindl. & Rosaceae & Nespereira & Exótica \\
\hline Erythrina indica var. picta B. \& M. & Fabaceae & Eritrina-rajada & Exótica \\
\hline Eucalyptus grandis W. Hill ex Maiden & Myrtaceae & Eucalipto & Exótica \\
\hline Eugenia cumini (L.) Druce & Myrtaceae & Jambolão & Exótica \\
\hline Eugenia jambolana & Myrtaceae & Jambo & Exótica \\
\hline Euphorbia leucocephala Lam. & Euphorbiaceae & Cabeça-de-velho & Exótica \\
\hline Euphorbia pulcherrima Willd. ex Klotzsch & Euphorbiaceae & Bico-de-papagaio & Exótica \\
\hline Ficus benjamina L. & Moraceae & Ficus-benjamim & Exótica \\
\hline Ficus elastica Roxb. & Moraceae & Seringueira-de-jardim & Exótica \\
\hline Ficus lyrata Warb. & Moraceae & Figueira & Exótica \\
\hline Ficus microcarpa L. f. & Moraceae & Ficus & Exótica \\
\hline Ficus variegata Blume & Moraceae & Ficus & Exótica \\
\hline Grevillea banksii R.Br. & Proteaceae & Grevilha-anã & Exótica \\
\hline Grevillea robusta A. Cunn. ex R. Br. & Proteaceae & Grevilha & Exótica \\
\hline Hibiscus rosa-sinensis L. & Malvaceae & Hibisco & Exótica \\
\hline Hovenia dulcis Thunb. & Rhamnaceae & Uva-japonesa & Exótica \\
\hline Jacaranda mimosifolia D. Don & Bignoniaceae & Jacarandá-mimoso & Exótica \\
\hline Jatropha gossypiifolia L. & Euphorbiaceae & Mamoninha & Exótica \\
\hline Koelreuteria paniculata Laxm. & Sapindaceae & Lanterna-japonesa & Exótica \\
\hline Lagerstroemia indica L. & Lythraceae & Resedá & Exótica \\
\hline Leucaena leucocephala (Lam.) de Wit & Fabaceae & Leucena & Exótica \\
\hline Ligustrum japonicum Thunb. & Oleaceae & Alfeneiro & Exótica \\
\hline Ligustrum lucidum W.T. Aiton & Oleaceae & Alfeneiro & Exótica \\
\hline Litchi chinensis Sonn. & Sapindaceae & Lichia & Exótica \\
\hline Livistona chinensis (Jacq.) R. Br. ex Mart. & Arecaceae & Palmeira-leque & Exótica \\
\hline Malpighia glabra L. & Malpighiaceae & Acerola & Exótica \\
\hline Mangifera indica L. & Anacardiaceae & Mangueira & Exótica \\
\hline Melaleuca leucadendra (L.) L. & Myrtaceae & Melaleuca & Exótica \\
\hline Melaleuca lineariifolia L. & Myrtaceae & Floco-de-neve & Exótica \\
\hline Melia azedarach L. & Meliaceae & Santa-bárbara & Exótica \\
\hline Michelia champaca L. & Magnoliaceae & Magnólia-amarela & Exótica \\
\hline Morus nigra L. & Moraceae & Amoreira & Exótica \\
\hline Murraya exotica L. & Rutaceae & Falsa-murta & Exótica \\
\hline Musa velutina $\mathrm{H}$. Wendl. \& Drude & Musaceae & Bananeira & Exótica \\
\hline Muntingia calabura L. & Muntingiaceae & Calabura & Exótica \\
\hline
\end{tabular}


ISERNHAGEN, I. ET AL.

\begin{tabular}{|c|c|c|c|}
\hline Nerium oleander $\mathrm{L}$. & Apocynaceae & Espirradeira & Exótica \\
\hline Persea americana Mill. & Lauraceae & Abacateiro & Exótica \\
\hline Pinus elliottii Engelm. & Pinaceae & Pinheiro & Exótica \\
\hline Plumeria rubra L. & Apocynaceae & Jasmim-manga & Exótica \\
\hline $\begin{array}{l}\text { Polyscias filicifolia (C. Moore ex E. Fourn.) L.H. } \\
\text { Bailey }\end{array}$ & Araliaceae & Árvore-da-felicidade & Exótica \\
\hline Prunus serrulata Lindl. & Rosaceae & Sakura & Exótica \\
\hline Espécie & Família & Nome Comum & Ocorrência \\
\hline Punica granatum $\mathrm{L}$. & Punicaceae & Romã & Exótica \\
\hline Radermachera sp. & Bignoniaceae & Radermaquera & Exótica \\
\hline Roystonea oleracea (Jacq.) O.F. Cook & Arecaceae & Palmeira Imperial & Exótica \\
\hline Roystonea regia (H.B.K.) O.F. Cook & Arecaceae & Palmeira Real & Exótica \\
\hline Salix babylonica L. & Salicaceae & Chorão & Exótica \\
\hline Schefflera actinophylla (Endl.) Harms & Araliaceae & Brassaia, cheflera & Exótica \\
\hline Senna siamea (Lam.) H.S. Irwin \& Barneby & Fabaceae & Cássia pequena & Exótica \\
\hline Spathodea campanulata P. Beauv. & Bignoniaceae & Espatódia & Exótica \\
\hline Syzygium cumini (L.) Skeels & Myrtaceae & Jambolão & Exótica \\
\hline Syzygium malaccense (L.) Merr. \& L.M. Perry & Myrtaceae & Jambo-vermelho & Exótica \\
\hline Tabebuia pentaphylla (L.) Hemsl. & Bignoniaceae & Ipê-rosa-americano & Exótica \\
\hline Tamarindus indica L. & Fabaceae & Tamarindo & Exótica \\
\hline Tecoma stans (L.) Juss. ex Kunth & Bignoniaceae & Ipê-mirim & Exótica \\
\hline Terminalia catappa L. & Combretaceae & Chapéu-de-sol & Exótica \\
\hline Thevetia peruviana (Pers.) K. Schum. & Apocynaceae & Chapéu-de-napoleão & Exótica \\
\hline Tipuana tipu (Benth.) Kuntze & Fabaceae & Tipuana & Exótica \\
\hline Thuia sp. & Pinaceae & Tuia & Exótica \\
\hline Yucca sp. & Agavaceae & luca & Exótica \\
\hline Anacardium occidentale L. & Anacardiaceae & Caju & Nativa \\
\hline Bertholletia excelsa Link & Fabaceae & Castanheira & Nativa \\
\hline Bixa orellana L. & Bixaceae & Urucum & Nativa \\
\hline Caesalpinia echinata Lam. & Fabaceae & Pau-Brasil & Nativa \\
\hline Caesalpinia peltophoroides Benth. & Fabaceae & Sibipiruna & Nativa \\
\hline Cassia ferruginea (Schrader) Schrader ex DC. & Fabaceae & $\begin{array}{r}\text { Canafístula-de- } \\
\text { besouro }\end{array}$ & Nativa \\
\hline Cecropia hololeuca Miq. & Urticaceae & Embaúba & Nativa \\
\hline $\begin{array}{l}\text { Cinnamomum burmannii (Nees \& T. Nees) } \\
\text { Blume }\end{array}$ & Lauraceae & Cinamomum & Nativa \\
\hline Cocos nucifera L. & Arecaceae & Coqueiro-da-bahia & Nativa \\
\hline Eugenia involucrata DC. & Myrtaceae & Cereja-do-rio-grande & Nativa \\
\hline Hibiscus tiliaceus L. & Malvaceae & Hibisco & Nativa \\
\hline Licania tomentosa (Benth.) Fritsch & Chrysobalanaceae & Oiti & Nativa \\
\hline Miconia cinnamomifolia (DC.) Naudin & Melastomataceae & Micônia & Nativa \\
\hline Pachira aquatica Aubl. & Malvaceae & Munguba & Nativa \\
\hline Psidium guajava L. & Myrtaceae & Goiabeira & Nativa \\
\hline Samanea saman (Jacq.) Merr. & Fabaceae & Samaneiro & Nativa \\
\hline Schinus molle L. & Anacardiaceae & Falso-chorão & Nativa \\
\hline Schinus terebinthifolia Raddi & Anacardiaceae & Aroeira-pimenteira & Nativa \\
\hline Schizolobium parahyba (Vell.) S.F. Blake & Fabaceae & Guapuruvu & Nativa \\
\hline Spondias purpurea L. & Anacardiaceae & Siriguela & Nativa \\
\hline Stifftia chrysantha Mikan & Asteraceae & Diadema & Nativa \\
\hline Tabebuia sp. & Bignoniaceae & Ipê & Nativa \\
\hline Theobroma cacao L. & Malvaceae & Cacaueiro & Nativa \\
\hline Tibouchina mutabilis Cogn. & Melastomataceae & Manacá-da-serra & Nativa \\
\hline Caesalpinia leiostachya (Benth.) Ducke & Fabaceae & Pau-ferro & Nativa \\
\hline
\end{tabular}


Trazendo a riqueza arbórea regional...

\begin{tabular}{|c|c|c|c|}
\hline Lafoensia glyptocarpa Koehne & Lythraceae & Mirindiba & Nativa \\
\hline Tibouchina granulosa (Desr.) Cogn. & Melastomataceae & Quaresmeira & Nativa \\
\hline Triplaris americana L. & Polygonaceae & Pau-formiga & Nativa \\
\hline Bauhinia forficata Link & Fabaceae & Pata-de-vaca & Nativa regional \\
\hline Cariniana legalis (Mart.) Kuntze & Lecythidaceae & Jequitibá & Nativa regional \\
\hline Cassia leptophylla Vogel & Fabaceae & Falso-barbatimão & Nativa regional \\
\hline Cedrela fissilis Vell. & Meliaceae & Cedro & Nativa regional \\
\hline Espécie & Família & Nome Comum & Ocorrência \\
\hline Ceiba speciosa (A. St.-Hil.) Ravenna & Malvaceae & Paineira & Nativa regional \\
\hline Enterolobium contortisiliquum (Vell.) Morong & Fabaceae & Orelha de nego & Nativa regional \\
\hline Erythrina mulungu Mart. ex Benth. & Fabaceae & Mulungu & Nativa regional \\
\hline Esenbeckia leiocarpa Engl. & Rutaceae & Guarantã & Nativa regional \\
\hline Eugenia brasiliensis Lam. & Myrtaceae & Grumixama & Nativa regional \\
\hline Eugenia pyriformis & Myrtaceae & Uvaia & Nativa regional \\
\hline Eugenia uniflora L. & Myrtaceae & Pitangueira & Nativa regional \\
\hline Holocalyx balansae Micheli. & Fabaceae & Alecrim-de-campinas & Nativa regional \\
\hline Hymenaea courbaril L. & Fabaceae & Jatobá & Nativa regional \\
\hline Inga uruguensis Hook. \& Arn. & Fabaceae & Ingá & Nativa regional \\
\hline Maytenus ilicifolia (Schrad.) Planch. & Celastraceae & Espinheira-santa & Nativa regional \\
\hline Myrciaria trunciflora O. Berg & Myrtaceae & Jaboticabeira & Nativa regional \\
\hline Myrocarpus frondosus Allemão & Fabaceae & Cabreúva & Nativa regional \\
\hline Nectandra megapotamica (Spreng.) Mez & Lauraceae & Canelinha & Nativa regional \\
\hline Peltophorum dubium (Spreng.) Taub. & Fabaceae & Canafistula & Nativa regional \\
\hline Plinia edulis (Vell.) Sobral & Myrtaceae & Cambucá & Nativa regional \\
\hline Pseudobombax grandiflorum (Cav.) A. Robyns & Malvaceae & Embiruçu & Nativa regional \\
\hline Psidium cattleianum Sabine & Myrtaceae & Araça & Nativa regional \\
\hline $\begin{array}{l}\text { Rustia formosa (Cham. \& Schltdl. ex DC.) } \\
\text { Klotzsch }\end{array}$ & Rubiaceae & Cafezinho-de-jardim & Nativa regional \\
\hline Sapindus saponaria L. & Sapindaceae & Sabão-de-soldado & Nativa regional \\
\hline $\begin{array}{l}\text { Senna macranthera (DC. ex Collad.) H.S. Irwin } \\
\text { \& Barneby }\end{array}$ & Fabaceae & Pau-fava & Nativa regional \\
\hline Solanum granuloso-leprosum Dunal & Solanaceae & Fumo bravo & Nativa regional \\
\hline Syagrus romanzoffiana (Cham.) Glassman & Arecaceae & Jerivá & Nativa regional \\
\hline Tabebuia avellanedae Lorentz ex Griseb. & Bignoniaceae & Ipê-roxo & Nativa regional \\
\hline Tabebuia chrysotricha (Mart. ex A. DC.) Standl. & Bignoniaceae & $\begin{array}{r}\text { Ipê-amarelo-do- } \\
\text { cerrado } \\
\end{array}$ & Nativa regional \\
\hline Tabebuia impetiginosa (Mart. ex DC.) Standl. & Bignoniaceae & $\begin{array}{r}\text { Ipê-roxo-de-bola- } \\
\text { pequeno } \\
\end{array}$ & Nativa regional \\
\hline Tabebuia roseoalba (Ridl.) Sandwith & Bignoniaceae & Ipê-branco & Nativa regional \\
\hline Tabebuia vellosoi Toledo & Bignoniaceae & Ipê-amarelo & Nativa regional \\
\hline Zeyheria tuberculosa (Vell.) Bureau & Bignoniaceae & Ipê felpudo & Nativa regional \\
\hline
\end{tabular}

Tomando como exemplo apenas o número potencial de espécies arbóreas existentes na Floresta Estacional Semidecidual, utilizou-se como base o trabalho de Ramos et al. (2008), que apresenta um total de 238 espécies para essa formação (para Piracicaba ver trabalhos citados em Rodrigues, 1999). Mesmo que sejam excluídas dessa lista potencial as árvores sem aptidão para arborização urbana e aquelas que não sejam encontradas regionalmente, o número de árvores nativas regionais encontradas nas três cidades aqui analisadas está muito aquém do que seria importante em um contexto regional. A Figura 1 
ilustra a proporção dos três grupos de ocorrência considerados no presente trabalho, comparando as cidades de Jaú, Bocaina e Piracicaba.

Ao se considerar também a proporção de indivíduos arbóreos utilizados nessas cidades, há uma nítida desproporção de freqüência entre os três grupos de ocorrência. Em Piracicaba, dos 6752 indivíduos amostrados, 3.984 (59\%) são de espécies exóticas, 2.074 $(30,72 \%)$ são de nativas e somente $694(10,28 \%)$ são de nativas regionais. Somente a espécie Murraya exotica L. (falsa-murta) compõe 16,88\% do total de indivíduos da cidade. Outras quatro espécies somadas à falsa-murta compõem aproximadamente 52\% do total de indivíduos da cidade, sendo duas exóticas (Nerium oleander L. - espirradeira, e Lagerstroemia indica L. - resedá) e duas nativas brasileiras (Caesalpinia peltophoroides Benth. - sibipiruna, e Licania tomentosa (Benth.) Fritsch - oiti). Além do fato de serem exóticas ao ecossistema regional, vale lembrar que as espécies falsa-murta, espirradeira e resedá não chegam a formar árvores de grande porte, sendo também questionável seu uso para fornecimento de benefícios como sombra. A falsa murta também compõe o maior percentual do total de indivíduos da cidade de Bocaina (29\%). Nesse censo, dos cinco setores nos quais a cidade foi dividida, em quatro a freqüência de ocorrência de indivíduos de duas ou três espécies supera os 50\%. Em Jaú o estudo da proporção dos indivíduos de diferentes espécies realizado em cada uma das 5 zonas em que a cidade foi dividida revelou padrões semelhantes, com a espécie exótica Ligustrum lucidum W.T. Aiton ocupando entre 30,68 a $46,52 \%$ do total de indivíduos plantados.

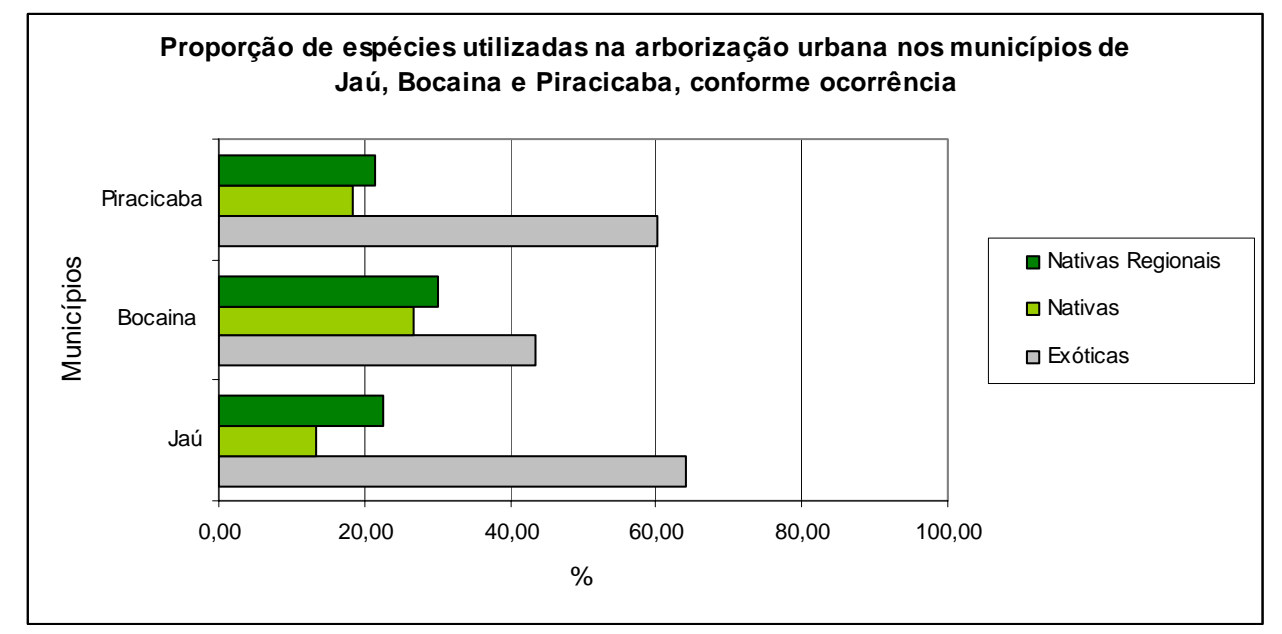

Figura 1: Proporção de espécies de diferentes ocorrências na arborização dos municípios de Piracicaba, Bocaina e Jaú, Estado de São Paulo (a partir de dados dos trabalhos de SOUZA et al., 2004; MARQUES, 2005 e Demóstenes da Silva Filho, dados não publicados).

Obviamente esse padrão não pode ser extrapolado para todas as cidades paulistas ou mesmo brasileiras sem uma avaliação mais completa, mas já dá uma idéia do balanço de riqueza de espécies e freqüência de indivíduos na região central do Estado de São Paulo. 
Mais inventários ou censos precisam ser realizados ou analisados para diagnosticar melhor essa situação nos centros urbanos do Brasil, mas é bem provável que o quadro demonstre um padrão semelhante, como sugerido por Silva (2008).

Embora vários dos trabalhos consultados ressaltem a importância da riqueza regional na composição das espécies utilizadas nas cidades, não foram encontrados estudos que indiquem qual seria uma proporção ideal entre as espécies de diferentes ocorrências. Uma abordagem sobre proporções na paisagem foi considerada nos estudos de Clarkson et al. (2007), que avaliaram a área de cobertura de ecossistemas nativos em círculos concêntricos que iam do núcleo urbano às áreas periurbanas ao redor de cidades da Nova Zelândia, em distância de até $20 \mathrm{Km}$ do centro. Com base em estudos de Biologia da Conservação, os autores consideraram como ideal uma cobertura mínima de $10 \%$ de áreas de vegetação nativa da região, recomendando a proteção desses ecossistemas naturais preferencialmente em áreas periurbanas, capazes de abrigar maiores extensões de área.

Apesar dessa abordagem parecer minimamente adequada para áreas naturais periurbanas, não se sabe se a proporção de $10 \%$ pode ser extrapolada para o número de indivíduos utilizados na arborização urbana. Sugere-se que esse número seja tão grande quanto possível, tanto em termos de riqueza de espécies como em abundância de indivíduos, desde que obviamente essas espécies atendam aos requisitos supracitados para uso na arborização em cidades. Porém, há que se realizar mais estudos para evitar o uso apenas do bom senso no processo decisório, visto que essa atitude pode dar margem a decisões deveras arbitrárias. Alguns parâmetros podem ser levados em consideração para embasar a proporção de uso de nativas regionais e exóticas, como a proximidade de remanescentes de vegetação nativa e a necessidade de criação de corredores ou trampolins ecológicos para promoção do trânsito de animais. No entorno e entre essas áreas o uso de espécies arbóreas nativas regionais deveria ser priorizado. A escolha das espécies pode também, por exemplo, estar relacionada ao grau de uso de espécies, especialmente pelas aves: espécies mais usadas por espécies de aves regionais seriam priorizadas, tanto em riqueza como em abundância.

\section{CONSIDERAÇÕES FINAIS}

Embora haja alguns exemplos pontuais de uso de espécies arbóreas nativas de ecossistemas brasileiros em cidades, ainda existe certo desconhecimento, ou mesmo despreocupação, sobre o uso de espécies adaptadas aos ecossistemas locais, aqui denominadas nativas regionais. Em parte isso pode ser atribuído a fatores culturais, e 
também ao fato de ainda não se conhecer o comportamento dessas espécies nos ambientes urbanos, reconhecidamente diferente do ambiente natural onde elas se desenvolvem mais adequadamente. Mais estudos precisam ser realizados para sanar essa lacuna de conhecimento na Silvicultura Urbana, se possível indicando aos administradores do espaço urbano uma proporção numérica mais precisa.

O presente trabalho não tem por intenção condenar o uso de espécies exóticas, nem indicar somente o uso de espécies nativas regionais na arborização urbana. A única ênfase é a contra-indicação expressa do uso de espécies exóticas invasoras, que não devem ser utilizadas (princípio da precaução) e, quando presentes, devem ser gradativamente erradicadas. Ações nesse sentido já vêm sendo adotadas pelo Poder Público, como pelo Instituto Ambiental do Paraná (IAP, 2007). No entanto, há que se considerar os benefícios do uso das espécies nativas regionais para o conjunto da biota regional, uma vez que essa poderia dispor de mais recursos aos quais evolutivamente estão mais adaptadas. Dependendo do grau de perturbação do ambiente, é possível inclusive que a própria regeneração natural seja capaz de restabelecer a cobertura vegetacional nativa, ação que pode ser manejada em parques ou terrenos ociosos da malha urbana. A proposta do presente trabalho é a valorização do potencial local, ainda mais se o local demonstrar tantos ou mais benefícios que o exótico.

O espaço urbano, embora possa se tornar mais permeável ao trânsito de espécies da biota regional, especialmente aves e morcegos, não será nunca equivalente ao ecossistema original. Dessa forma, políticas de implantação de arborização urbana, baseadas em espécies arbóreas nativas regionais, devem ser relacionadas a ações de conservação da natureza de escala regional, criando verdadeiros mosaicos de situações que promovam a sobrevivência da biota regional e o bem-estar das populações humanas.

O uso das espécies nativas regionais também deve levar em consideração questões culturais. A introdução de exóticas está normalmente associada a um senso de nostalgia de colonizadores em relação à sua terra natal, conforme revisão apresentada por Stewart et al. (2004). Essa barreira cultural deve ser abordada e transposta nas campanhas de convencimento e valorização da flora regional. Em revisão apresentada por Miller (2006), vários pesquisadores sugerem que uma das causas da homogenização biótica é a falta de contato dos seres humanos com a riqueza biológica, à medida que a maior parte das pessoas habita ambientes urbanos. Não só os encontros com a natureza têm ficado raros, mas a qualidade desses encontros também tem se deteriorado. Uma das possibilidades de reverter esse processo, com impacto acentuado na melhoria de qualidade de desenvolvimento emocional e intelectual de crianças, é aproximar as pessoas da riqueza biológica no próprio lugar onde elas atualmente vivem (Turner et al. 2004). A utilização da riqueza de árvores nativas regionais pode possibilitar esse processo, uma vez que seu uso 
pode estar associado a um aumento de freqüência de ocorrências de outras espécies associadas, especialmente da ornitofauna (Savard et al. 2000), grupo animal que normalmente possui grande aceitação popular. Alcançar esse objetivo demandará uma intensa colaboração entre cientistas e administradores do espaço urbano, sendo importante a comunicação e o envolvimento com a população local. Como é usual em alguns planejamentos de arborização urbana, há que se realizar pesquisas sobre a aceitação das nativas regionais por parte da população.

Sugere-se também que, nos mecanismos de valoração econômica na arborização urbana, o fato da árvore ser de uma espécie nativa regional gere aumento no respectivo valor. Esse valor deve ser tanto maior quanto mais rara for a espécie na arborização, mais difícil a obtenção de mudas e maior o grau de ameaça de extinção regional. Também será necessário fomentar a reestruturação dos viveiros municipais para incorporar maior riqueza de espécies arbóreas regionais, em quantidade adequada para atender à demanda. Os prestadores de serviços de manutenção deverão ser instruídos a respeito das características dessas espécies.

Se após plantios experimentais forem atendidos os requisitos para uso das espécies nativas regionais, há motivos suficientes e importantes para a utilização dessas árvores nas cidades, e os autores acreditam e defendem que o uso dessas trará somente benefícios em escala local e regional.

\section{AGRADECIMENTOS}

Os autores gostariam de agradecer ao Eng. Agrônomo Pedro H.S. Brancalion, pela revisão da classificação das espécies nas tabelas 1, 2 e 3.

\section{REFERÊNCIAS BIBLIOGRÁFICAS}

ALVEY, A.A. Promoting and preserving biodiversity in the urban forest. Urban Forestry \& Urban Greening, n.5, p. 195-201, 2005.

BIONDI, D.; LEAL, L. Caracterização das plantas produzidas no Horto Municipal da Barreirinha - Curitiba / PR. Rev. SBAU, Piracicaba, v.3, n.2, p. 20-36, jun. 2008.

BLUM, C.T.; BORGO, M.; SAMPAIO, A.C.F. Espécies exóticas invasoras na arborização de vias públicas de Maringá-PR. Rev. SBAU, Piracicaba, v.3, n.2, p. 78-97, jun. 2008.

BORTOLETO, S. et al. Composição e distribuição da arborização viária da Estância de Águas de São Pedro - SP. Rev. SBAU, v.2, n.3, p.32-46, 2007.

BRUN, F.G.K.; LINK, D.; BRUN, E.J. O emprego da arborização na manutenção da biodiversidade de fauna em áreas urbanas. Rev. SBAU, Piracicaba, v.2, n.1, p. 117-127, 2007. 
CLARKSON, B.D.; WEHI, P.M.; BRABYN, L.K. A spatial analysis of indigenous cover patterns and implications for ecological restoration in urban centres, New Zealand. Urban Ecosyst, n.10, p.441-457, 2007.

COLETTO, E.P.; MULLER, N.G.; WOLSKI, S.S. Diagnóstico da arborização das vias públicas do Município de Sete de Setembro - RS. Rev. SBAU, v.3, n.2, p.110-122, 2008.

CRESTANA, M.S.M.; SILVA FILHO, D.F.; BERTONI, J.E.A.; GUARDIA, J.F.C.; ARAUJO, R.T.A. Arvores e Cia. Campinas: CATI, 2007. 132 p.

DANTAS, I.C.; SOUZA, C.M.C. Arborização urbana na cidade de Campina Grande - PB: Inventário e suas espécies. Revista de Biologia e Ciências da Terra, v.4, n.2, 2004.

FARIA, J.L.G.; MONTEIRO, E.A.; FISCH, S.T.V. Arborização de vias públicas no município de Jacareí - SP. Rev. SBAU, v.2, n.4, p.20-33, 2007.

FORMAN, R.T.T.; GODRON, M. Landscape Ecology. New York: John Wiley \& Sons, 1986.

GARDEN, J.C.; MCALPINE, C.A; POSSINGHAM, H.P.; JONES, D.N. Habitat structure is more important than vegetation composition for local-level management of native terrestrial reptile and small mammal species living in urban remnants: A case study from Brisbane, Austrália. Austral Ecology, n.32, p.669-685, 2007.

HARRIS, R.W.; CLARK, J.R.; MATHENY, N.P. Arboriculture: integrated management of landscape trees, shrubs, and vines. New Jersey: Prentice Hall, 1999.

IAP - Instituto Ambiental do Paraná. Portaria IAP nº 095, de 22 de maio de 2007. Disponível http://www.iap.pr.gov.br/arquivos/File/iap/port 95 07.pdf. Acesso em 09/05/2009.

LAZARO, I; SALES FILHO, M.A.; TEJIDO, A.L.; FERREIRA, S.N.M. Guia de Arborização Urbana. $2002 . \quad$ Disponível 2 http://www.coelba.com.br/ARQUIVOS EXTERNOS/ENERGIA\%20SOCIAL\%20E\%20AMBIE NTAL/MEIO\%20AMBIENTE/PROJETOS\%20AMBIENTAIS\%20ESPECIAIS/arborizacao urb ana.pdf. Acessado em 28/09/2008.

INSTITUTO FLORESTAL. Reserva da Biosfera do Cinturão Verde da Cidade de São Paulo. Disponível em: http://www.iflorestsp.br/rbcv/. Acessado em 06.10.2008.

JIM, C.Y. Green-space preservation and allocation for sustainable greening of compact cities. Cities, v.21, n.4, p.311-320, 2004.

KENWORTHY, J.R. The eco-city: ten key transport and planning dimensions for sustainable city development. Environment and Urbanization, v.18, n.1, p.67-85, april 2006.

LORENZI, H. Árvores brasileiras: manual de identificação e cultivo de plantas arbóreas nativas do Brasil. Nova Odessa: Plantarum, 1992.

LORENZI, H. Árvores brasileiras: manual de identificação e cultivo de plantas arbóreas nativas do Brasil. 2. ed. Nova Odessa: Plantarum, 1998. v.2.

MACHADO, R.R.B.; MEUNIER, I.M.J.; SILVA, J.A.A.; CASTRO, A.A.J.F. Árvores nativas para a arborização de Terezina, Piauí. Rev. SBAU, v.1, n.1, 2006. p.10-18.

MARQUES, A.L. (coord.). Relatório Final do Inventário da Arborização Urbana do Município de Bocaina/SP. Bocaina: Instituto Internacional de Ecologia / Diretoria de Agricultura e Meio Ambiente da Prefeitura Municipal de Bocaina, 2005.

MARTO, G.B.T.; BARRICHELO, L.E.G.; SILVA FILHO, D.F.; MULLER, P.H. Arborização Urbana. 2006.2 Disponível em: http://www.infobibos.com/Artigos/ArborizacaoUrbana/ArborizacaoUrbana.htm. Acessado em 28/09/2008. 
Trazendo a riqueza arbórea regional...

MCDONALD, R.I.; KAREIVA, P.; FORMAN, R.T.T. The implications of current and future urbanization for global protected areas and biodiversity conservation. Biological Conservation, v. 141, p. 1695-1703, 2008.

MILLER, J.M. Restoration, reconciliation, and reconnecting with nature nearby. Biological Conservation, v.127, p.356-361, 2006.

MILLER, R.W. Urban Forestry: planning and managing urban greenspaces. $2^{\text {nd }}$ ed. New Jersey: Prentice-Hall, 1997.

MOBOT - MISSOURI BOTANICAL GARDEN - Vascular Tropics nomenclatural data base. Disponível em: http://mobot.mobot.or/W3T/Search/vast.html/. Acessado em 07.10.2008.

NICOLINI, E. M. Composição florística e estrutura fitossociológica do estrato arbóreo em mata mesófila semidecídua no município de Jaú-SP. Dissertação de Mestrado. Universidade Estadual Paulista. Rio Claro, 1990. 179p.

OLIVEIRA, C.A. de; SANTOS, C.J.F. Florestas urbanas: normas de uso e ocupação do solo para proteção de unidades de conservação nas cidades. In: MILANO, M.S. (coord.). CONGRESSO BRASILEIRO DE UNIDADES DE CONSERVAÇÃO, 4, 2004, Curitiba. Anais... Curitiba: Fundação O Boticário de Proteção à Natureza, Rede Nacional PróUnidades de Conservação, 2004. p. 542-549.

PENNINGTON, D.N.; HANSEL, J.; BLAIR, R.B. The conservation value of urban riparian areas for landbirds during spring migration: land cover, scale, and vegetation effects. Biological Conservation, n.141, p.1235-1248, 2008.

PIRES, N.A.M.T.; MELO, M.S.; OLIVEIRA, D.E.; XAVIER-SANTOS, S. Diagnóstico da Arborização Urbana do Município de Goiandira, Goiás. Revista Brasileira de Biociências, v. 5, supl. 1, p. 537-539, 2007.

PIVETTA, K.F.L.; SILVA FILHO, D.F. Arborização Urbana. Boletim Acadêmico. Serie Arborização Urbana. UNESP/FCAV/FUNEP. Jaboticabal, 2002. 74 p.

PMB - Prefeitura Municipal de Bauru. Disponível em: http://www.bauru.sp.gov.br/prefeitura/pmb.php?cat=8\&action=ler\&news id=213\&acao=cat.

Acessado em 02.10.2008.

RAMOS, V.S.; DURIGAN, G.; FRANCO, G.A.D.C.; SIQUEIRA, M.F. de; RODRIGUES, R.R. Árvores da Floresta Estacional Semidecidual - guia de identificação de espécies. São Paulo: Edusp / Biota-Fapesp, 2008. 320p.

REIS, A.; ANJOS, A. dos; BECHARA, F.C. Critérios para a seleção de espécies na arborização urbana ecológica. Sellowia, n. 53-55, p. 51-67, maio 2003.

RODRIGUES, R.R. A vegetação de Piracicaba e municípios do entorno. Circular Técnica IPEF, Piracicaba, n.189, ago. 1999.

ROSSATTO, D.R. TSUBOY, M.S.F.; FREI, F. Arborização urbana na cidade de Assis-SP: uma abordagem quantitativa. Revista SBAU, v.3, n.3, 2008, p. 1-16.

SAVARD, J.L.; CLERGEAU, P.; MENNECHEZ, G. Biodiversity concepts and urban ecosystems. Landscape and Urban Planning, v.48, p. 131-142, 2000.

SILVA, L.M. Reflexões sobre a identidade arbórea das cidades. Rev. SBAU, Piracicaba, v.3, n.3, p. 65-71, set. 2008.

SOUSA, C.A. de. Turning brownfields into green space in the City of Toronto. Landscape and Urban Planning, v.62, n.4, p.181-198, 2003.

SOUZA, A.M. de; NACHTERGAELE, M.F.; CARBONI, M. Inventário Florestal da arborização do município de Jaú/SP. Jaú: Instituto Pró-Terra \& Secretaria do Meio Ambiente - SEMEIA, 2004. Relatório técnico. 
SOUZA, V.C.; LORENZI, H. Botânica Sistemática: guia ilustrado para identificação das famílias de Angiospermas da flora brasileira, baseado em APG II. Nova Odessa: Plantarum, 2005. 640p.

STEWART, G.H.; IGNATIEVA, M.E.; MEURK, C.D.; EARL, R.D. The re-emergence of indigenous forest in na urban environment, Christchurch, New Zealand. Urban For. Urban Green., v.2, p.149-158, 2004.

ZERBE, S.; MAURER, U.; SCHMITZ, S.; SUKOPP, H. Biodiversity in Berlin and its potential for nature conservation. Landscape and Urban Planning, n.62, p. 139-148, 2003. 